\title{
Inferring the Spatial Distribution of Physical Activity in Children Population from Characteristics of the Environment
}

\author{
Ioannis Sarafis, Christos Diou, Vasileios Papapanagiotou, Leonidas Alagialoglou and Anastasios Delopoulos
}

\begin{abstract}
Obesity affects a rising percentage of the children and adolescent population, contributing to decreased quality of life and increased risk for comorbidities. Although the major causes of obesity are known, the obesogenic behaviors manifest as a result of complex interactions of the individual with the living environment. For this reason, addressing childhood obesity remains a challenging problem for public health authorities. The BigO project (https://bigoprogram.eu) relies on large-scale behavioral and environmental data collection to create tools that support policy making and intervention design. In this work, we propose a novel analysis approach for modeling the expected population behavior as a function of the local environment. We experimentally evaluate this approach in predicting the expected physical activity level in small geographic regions using urban environment characteristics. Experiments on data collected from 156 children and adolescents verify the potential of the proposed approach. Specifically, we train models that predict the physical activity level in a region, achieving $81 \%$ leave-one-out accuracy. In addition, we exploit the model predictions to automatically visualize heatmaps of the expected population behavior in areas of interest, from which we draw useful insights. Overall, the predictive models and the automatic heatmaps are promising tools in gaining direct perception for the spatial distribution of the population's behavior, with potential uses by public health authorities.
\end{abstract}

\section{INTRODUCTION}

The obesity epidemic soared in the recent decades, affecting a significant percentage of the global population. Obesity is increasing rapidly in the younger population, with the World Health Organization (WHO) estimating that over $18 \%$ of the children and adolescent population aged 5-19 were overweight or obese in 2016, compared to $4 \%$ in 1975 [1].

At the individual's level, the major cause of obesity is the imbalance between energy expenditure and energy intake [2]. However, behind this imbalance, there are complex behavior patterns and interactions associated with the living environment [3]. To this end, public health authorities are tasked with designing policies that create "active environments" and promote healthier lifestyle for the population [4], [5].

Towards this goal, the BigO: Big Data Against Childhood Obesity* project takes advantage of new data sources to create novel tools for policy making and intervention design [6], [7]. BigO relies on: 1) large-scale, objective monitoring

\footnotetext{
*The work leading to these results has received funding from the European Community's Health, demographic change and well-being Programme under Grant Agreement No. 727688 (https://bigoprogram.eu) 01/12/2016 - 30/11/2020. The Institution's Ethical Review Board approved all experimental procedures involving human subjects.

All authors are with Multimedia Understanding Group, Information Processing Laboratory, Aristotle University of Thessaloniki, Greece.
}

of the population's behavior, in real-life conditions, using commodity devices (e.g., smartphones and smartwatches); 2) estimating the environment characteristics using open and online data sources; and 3) associating population behaviors with environment characteristics to create actionable knowledge for public health authorities. An important aspect of the $\mathrm{BigO}$ methodologies is that the data are anonymized and aggregated in geographical regions in order to increase privacy protection for the participating individuals.

In this work, we present an analysis approach developed in the context of $\mathrm{BigO}$ for predicting behaviors in children and adolescent population using measurements of the environment. Then, we demonstrate the proposed approach in building models for predicting the expected physical activity level. The predictive models use measurements of the urban environment in relatively small geographic regions (approx. $0.57 \mathrm{~km}^{2}$ ) and predict the expected physical activity level for the resident population, as High or Low level.

Experiments are conducted on data collected from 156 children and adolescents during $\mathrm{BigO}$ pilots in the Thessaloniki metropolitan area. For each participating individual, we use accelerometry data to infer indicators of physical activity and GPS location data to identify the region of residence. In this experimental setup, the proposed approach achieves $81 \%$ leave-one-out accuracy, with high precision and recall for both classes.

Then, we demonstrate an application of the models in visualizing heatmaps of the predicted population behavior for areas of interest. The examination of the example heatmaps, in conjunction with the environment characteristics, reveals useful and actionable insights. The predictive models can also be directly adopted in urban or policy design, especially for simulating imminent changes in the population behavior as the outcome of possible policies and interventions.

The rest of the paper is organized as follows. First, Section III presents an overview of the proposed approach. Then, Section III demonstrates an experimental application for predicting the physical activity level for children and adolescent population. Section IV] uses the predictive models to generate heatmaps of the expected population behavior and provides a discussion using the visualizations. Finally, Section $\mathrm{V}$ concludes the paper.

\section{PROPOSED APPROACH}

Figure 1 sketches the proposed approach. The goal is to build predictive models that map local attributes of the environment to behaviors exhibited by the resident population. 
This is accomplished through the following steps:

1) Large-scale behavioral data collection in real-life conditions from the target population. We rely on commonly available sensors in smartphones and smartwatches, such as GPS and triaxial accelerometer. All behavioral data are recorded in real life, with no supervision. A smartphone application is used to collect and transmit the raw sensory data or calculated products.

2) Environment data collection from external data sources. For example, data from GIS, such as the location and type for Points of Interests (POIs), the availability of open spaces, etc., or data from statistical authorities (e.g. unemployment rate, income).

3) For each participating individual, we extract behavioral indicators from the collected sensory data. In this context, a behavioral indicator is an objectively measurable quantity for the behavior of one individual. For example, the average daily activity counts/min, the total steps, the types of the visited POIs, etc.

4) For each participating individual, we also identify the area of residence. For privacy protection, the residence location is assigned to a geohash [8]. Geohash is a geocode system where geographic locations are mapped to a grid of rectangular areas. An advantage of this encoding is that we can easily tune the size of the rectangles by changing the hash length (shorter hashes outline larger areas); thus, enforcing the desired level of privacy protection.

5) We calculate behavioral attributes for each geohash, which are aggregations for the behavioral indicators of the individuals living in the geohash. For example, the average of the activity counts/min indicator, the average daily steps, or the average number of weekly visits to fast food restaurants.

6) We calculate environmental attributes for each geohash (also termed "Local Extrinsic Conditions (LECs)" in BigO system [6]), using the available environment data. For example, the number of athletics facilities in the geohash, the number of fast food restaurants, or the local unemployment rate, etc.

7) Finally, we build predictive models that map environmental attributes to one or more behavioral attributes. The premise is that there exist known, but unquantifiable, causal relationships between the average (expected) population behavior and the local environment.

\section{EXPERIMENTAL APPLICATION}

This section demonstrates an experimental application of the proposed approach. The models predict the behavioral attribute "expected physical activity level" for relatively small geographic areas of residence. The predicted classes are in High or Low level. A set of urban environmental attributes is used as input to the models.

\section{A. Data and experimental setup}

Table I summarizes the data used in the experiments. The data were collected from 410 children and adolescents (ages

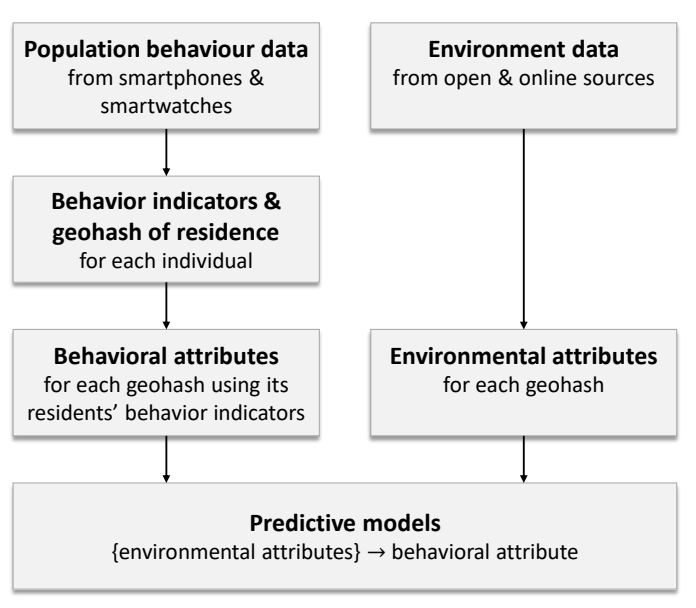

Fig. 1. Schematic illustration of the proposed approach

TABLE I

OVERVIEW OF EXPERIMENT DATA

\begin{tabular}{lr}
\hline Total number of children & 410 \\
Total hours of recordings & 90890 \\
Number of children passing selection criteria & 156 \\
Male / Female / Other - Not specified & $85 / 70 / 1$ \\
Number of unique geohashes of residence (length 6) & 42 \\
\hline
\end{tabular}

9-18) in the Thessaloniki metropolitan region, Greece. The participants contributed data using the "myBigO" app [9] in the context of school activities, under real-life conditions and without supervision during the data collection period. The "myBigO" app collects self-reported and sensory data (accelerometry and GPS location) from the smartphone and, if available, a paired smartwatch [9]. Typically, the data collection period is 1-2 weeks for each participating individual.

We applied two criteria to select the individuals for the experiments. First, we selected the ones for which we were able to clearly identify the geohash of the residence using heuristics ${ }^{1}$ Second, we selected only individuals with more than 20 hours of active recording sessions spanning across at least 3 different days. As an active recording session we consider when the individual is carrying the smartphone or wears a paired smartwatch and, thus, extraction of physical activity indicators is possible.

From the 410 participants in the Thessaloniki metropolitan region, 156 satisfied the selection criteria. The locations of residence were found in 42 different geohashes, using a 6 characters length 2 For each individual, we extracted the target behavioral indicator. In this experimental application, we calculated the average activity counts per minute [10] as the indicator of physical activity, using the collected accelerometry data of the active recording sessions.

Then, for each geohash with residents, we averaged its residents' behavioral indicators to calculate the behavioral attribute. Thus, the behavioral attribute is the expectation of the population behavior in each geohash. The probability distribution of the behavioral attribute is shown in Figure 2.

\footnotetext{
${ }^{1}$ Based on GPS data recorded in the hours 23:00 to 07:00.

${ }^{2}$ Approximately $610 \mathrm{~m} \times 930 \mathrm{~m}$ for Thessaloniki.
} 


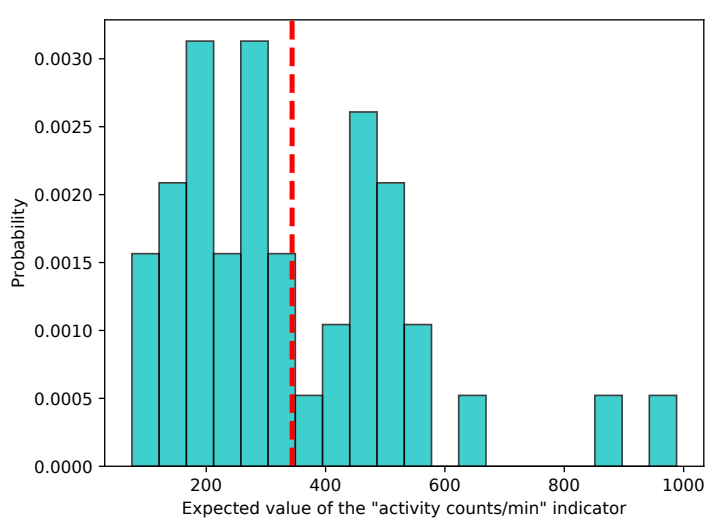

Fig. 2. Histogram of the behavioral attribute for the geohashes. The dashed line indicates the distribution mean (approx. 343.9 activity counts/min)

Finally, for each geohash, we calculated a set of environmental attributes from characteristics of the local urban environment. Specifically, each environmental attribute corresponds to the number of a specific POI type that is available in the geohash. We calculated 4 environmental attributes: "athletics and sports," "fast food and take away restaurants," "public parks" and "cafés and café bars." This information is provided from a variety of online data sources, such as OpenStreetMap, Google Maps, Foursquare, and Bing Maps.

\section{B. Model training}

We transformed the data to create a data set suitable for training binary classification algorithms. For this purpose, the $i$-th geohash, $i=1 \ldots 42$, was converted into the data point $\left(\mathbf{x}_{i}, y_{i}\right)$ that consists of:

- A 4-dimensional feature vector $\mathbf{x}_{i}$ that contains the environmental attributes. Each dimension is separately normalized in the range $[0,1]$.

- A binary class label $y_{i}$, where: $y_{i}=$ High if behavioral attribute is greater than the distribution mean; $y_{i}=$ Low otherwise. The mean value of the distribution is approximately 343.9 activity counts/min (see Figure 2).

The predictive models are based on the random forests (RF) algorithm [11]. Through a 10-fold cross-validation, we selected the optimal hyperparameters for the number of decision trees, the maximum number of levels in each tree and the measurement criterion for the quality of split.

\section{Results}

Using the RF algorithm and the optimal hyperparameters, we applied a leave-one-out (LOO) procedure to evaluate the prediction performance. During LOO, one geohash (from the 42 ones with residents) was left out, an RF model was trained using the rest of the geohashes, and the model's prediction was evaluated on the left-out geohash.

Table II shows the confusion matrix produced from the LOO procedure and Table III shows the corresponding classification metrics. The predictive models achieved high leave-one-out accuracy of $81 \%$. Furthermore, high precision and recall was demonstrated for both classes.
TABLE II

CONFUSION MATRIX FOR THE LOO PROCEDURE. ACCURACY $=\mathbf{8 1} \%$

\begin{tabular}{cc|cc} 
& \multicolumn{3}{c}{ Predicted } \\
& & Low & High \\
\hline \multirow{2}{*}{ Low } & 21 & 3 \\
High & 5 & 13
\end{tabular}

TABLE III

CLASSIFICATION METRICS

\begin{tabular}{llll}
\hline Class & Precision & Recall & F1-score \\
\hline Low & 0.81 & 0.88 & 0.84 \\
High & 0.81 & 0.72 & 0.76 \\
\hline
\end{tabular}

Overall, the results verify that it is feasible to build accurate generalizations for the population behavior in areas where only environmental data are available.

\section{HEATMAP VISUALIZATIONS}

In this section we exploit the high accuracy of the predictive models to generate heatmaps of the expected population behavior in target areas of interest.

Figure 3 shows the model predictions per geohash for a large part of the Thessaloniki metropolitan area. It is worth noting that the original data span over 42 geohashes, which is a relatively small percentage of the total area of interest in the

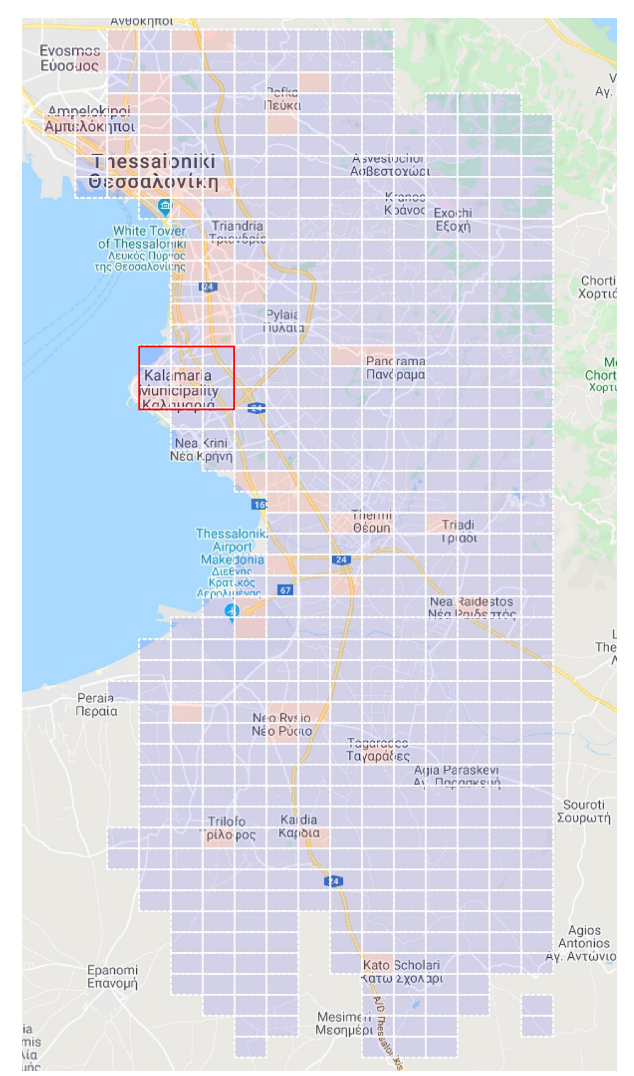

Fig. 3. Example heatmap for an area in the Thessaloniki metropolitan area. Grid is made from geohashes of hash length 6 . Geohashes predicted in the High class are colored red; geohashes predicted in the Low class are colored blue. The area marked with a red square is shown in detail in Figure 4 Map data: Google. 


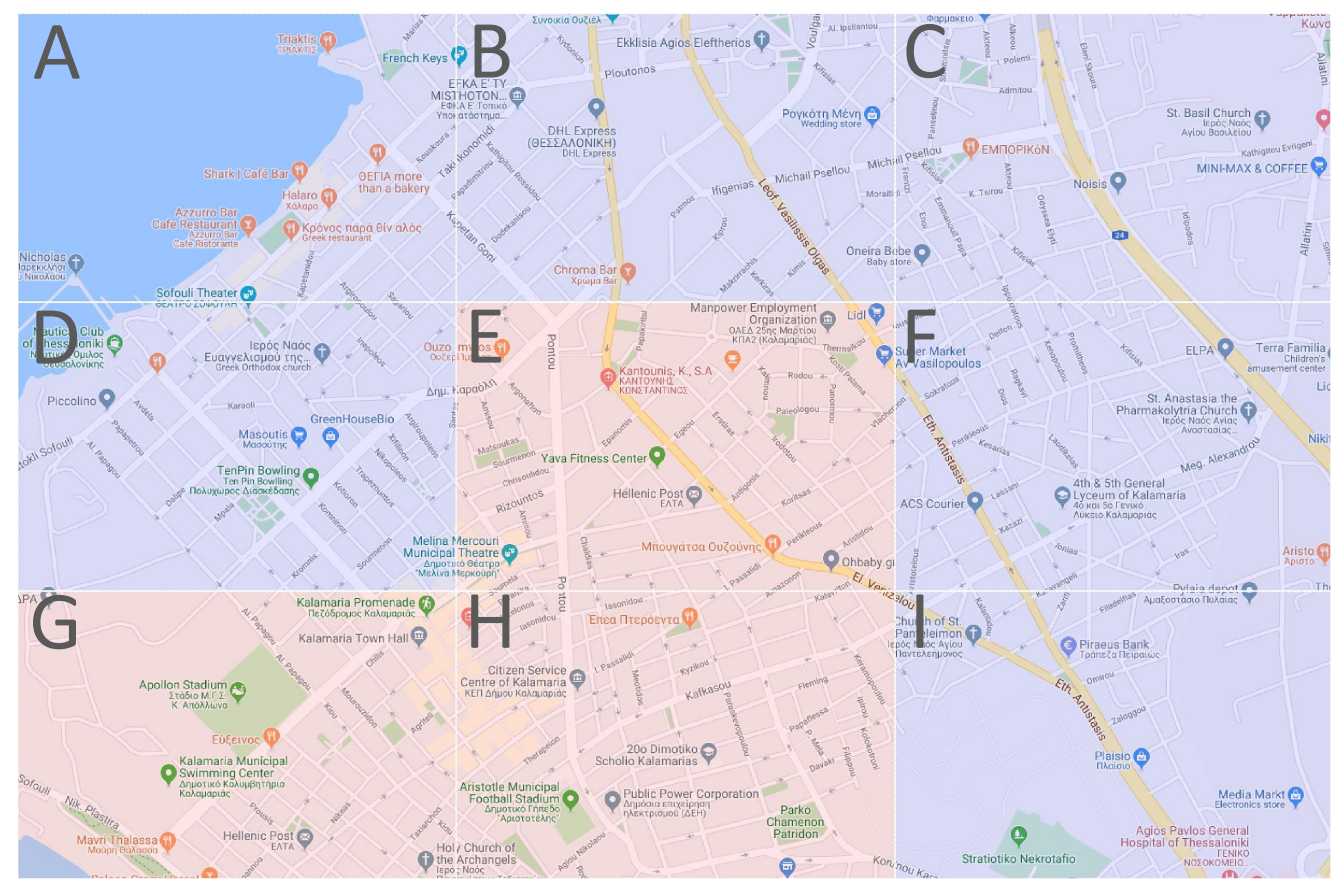

Fig. 4. Example heatmap for a municipality region in Thessaloniki city. The figure shows 9 geohashes (hash length 6 ). Geohashes predicted in the High class are colored red; geohashes predicted in the Low class are colored blue. Map data: Google.

Thessaloniki metropolitan region. Thus, using an imputation mechanism - such as the proposed predictive models — is the only way we can visualize complete and usable heatmaps.

Figure 4 shows a more targeted heatmap that covers an area in a municipality. The geohashes $\mathrm{E}, \mathrm{G}$ and $\mathrm{H}$ are predicted in the High level class, which is colored red in the heatmap. In these geohashes, there is a significant number of athletics/sports facilities and public parks, providing exercise and active recreation opportunities for the children and adolescents. Also, these areas have an increased number of food outlets and cafés, which is an indication of commercial and/or popular areas with pedestrian streets and squares where people walk.

On the other hand, such urban environment characteristics are not prominent in the other geohashes (A, B, C, D, F and I). These geohashes are predicted in the Low level class, colored blue in the heatmap. For example, geohash $\mathrm{F}$ is a fairly densely populated area, however, it lacks POIs that would contribute to an active lifestyle. Thus, from a public health authority's point-of-view, such heatmaps provide direct indications for areas in need of attention.

\section{CONCLUSIONS}

We presented a novel approach for building models that predict the expected population behavior from the local environment in the regions of residence. The proposed approach was demonstrated and evaluated in the application of predicting the expected physical activity level for children and adolescents population, using characteristics of the urban environment as input. The experiment data were collected in the context of BigO pilots from 156 children and adolescents in the Thessaloniki metropolitan region, Greece. The results verified the feasibility of the proposed approach. In addition, we demonstrated an application of the predictive models for visualizing heatmaps of the population behavior, from which we derived useful insights. The proposed approach can support further applications in urban design and policy making, for example in simulating the effects of policies and interventions on the population; however, further validation is needed in these research directions.

\section{REFERENCES}

[1] World Health Organisation, "Obesity and overweight, fact sheet," 2016, https://www.who.int/news-room/fact-sheets/detail/ obesity-and-overweight Last accessed on 2020-01-22.

[2] N. F. Butte, E. Christiansen, and T. I. A. Srensen, "Energy imbalance underlying the development of childhood obesity," Obesity, vol. 15, no. 12, pp. 3056-3066, 2007.

[3] I. Romieu et al., "Energy balance and obesity: what are the main drivers?" Cancer Causes \& Control, vol. 28, no. 3, pp. 247-258, 2017.

[4] World Health Organization, Global action plan on physical activity 2018-2030: more active people for a healthier world, 2019.

[5] B. Giles-Corti et al., "City planning and population health: a global challenge," The Lancet, vol. 388, no. 10062, pp. 2912 - 2924, 2016.

[6] C. Diou et al., "A methodology for obtaining objective measurements of population obesogenic behaviors in relation to the environment," Statistical Journal of the IAOS, vol. 35, no. 4, pp. 677 - 690, 2019.

[7] I. Sarafis, C. Diou, and A. Delopoulos, "Behaviour profiles for evidence-based policies against obesity," in 201941 st Annual International Conference of the IEEE Engineering in Medicine and Biology Society (EMBC), July 2019, pp. 3596-3599.

[8] G. M. Morton, "A computer oriented geodetic data base and a new technique in file sequencing," IBM Research, 1966.

[9] C. Maramis et al., "Developing a novel citizen-scientist smartphone app for collecting behavioral and affective data from children population," in 8th EAI International Conference on Wireless Mobile Communication and Healthcare, November 2019.

[10] W. W. Tryon and R. Williams, "Fully proportional actigraphy: A new instrument," Behavior Research Methods, Instruments, \& Computers, vol. 28, no. 3, pp. 392-403, Sep 1996.

[11] L. Breiman, "Random forests," Machine Learning, vol. 45, no. 1, pp. 5-32, Oct 2001. 